\title{
Performance Comparisons of External Modulated Hybrid Analog-Digital Signals in Electrical and Optical Domains
}

\author{
Wei-Ren Peng, Peng-Chun Peng, Yu-Ting Hsueh, Kai-Ming Feng, and Sien Chi
}

\begin{abstract}
A multipurpose network which integrates the functionalities of single radio channel and baseband data distribution has been proposed recently. In this letter, we further propose and compare two possible combination schemes which combine multiple radio channel and single digital baseband data in electrical and optical domains. Linearity of multiple radio channels and performance of digital baseband data are studied in this externally modulated hybrid analog-digital link. It is found that signals combined in the optical domain, while with higher cost, will outperform that in the electrical domain on the carrier-to-noise and distortion ratio. In addition, the baseband data performance is also improved with optical combining scheme since the optimum required modulation index is lower.
\end{abstract}

Index Terms-Carrier-to-noise and distortion ratio (CNDR), hybrid analog-digital transmission, Mach-Zehnder modulator (MZM), radio-over-fiber (RoF).

\section{INTRODUCTION}

$\mathbf{I}$ N RECENT years, both demands on wireless and wired line capacity are increasing. Facing the huge and increasingly growing capacity, we use the fiber as the transmission medium. The wireless signal suffers the insufficient bandwidth and serious loss problems with the coaxial cable. Owing to the broad bandwidth and lower loss of optical fiber, radio-over-fiber (RoF) which directly modulates the analog wireless signal on the optical carrier was proposed [3]. At the same time, the gradually matured fiber-to-the-home, curb, or building (FTT-x) networks are also under development. Since the high cost of distributed infrastructure of each network will be an obstacle to the carriers, it requires a good solution to merge the two distribution networks into one. A hybrid lightwave transmission system for subcarrier multiplexed video and broad-band integrated digital services networks with direct modulation scheme are proposed in [4]. External modulations on the hybrid high-speed analog-digital modulation are proposed and the interchannel interference between the analog and digital signal is also discussed [1], [2].

\footnotetext{
Manuscript received June 30, 2005; revised July 22, 2005. This work was supported in part by the National Science Council of R.O.C. under Contract NSC 93-2752-E-009-009-PAE, NSC 93-2215-E-155-005, NSC 93-2215-E-155-004.

W.-R. Peng, P.-C. Peng, and Y.-T. Hsueh are with the Department of Photonics and Institute of Electro-Optical Engineering, National Chiao-Tung University, Hsinchu, Taiwan 300, R.O.C. (e-mail: pwr.eo92g@nctu.edu.tw).

K.-M. Feng is with the Institute of Communication Engineering, National Tsing-Hua University, Hsinchu, Taiwan, 300, R.O.C.

$\mathrm{S}$. Chi is with the Department of Photonics and Institute of Electro-Optical Engineering, National Chiao-Tung University, Hsinchu, Taiwan 300, R.O.C. $\mathrm{He}$ is also with the Department of Electrical Engineering, Yuan-Ze University, Chung Li, Taiwan 320, R.O.C.

Digital Object Identifier 10.1109/LPT.2005.858152
}

However, no discussion is on the linearity of multiple radio channels among these proposed schemes and the problem of interchannel interference should be solved.

In this letter, we propose and demonstrate two possible schemes combining radio and baseband signals in electrical and optical domains. Linearity of multiple radio channels and performance of digital baseband data are compared between the two schemes. It is found that the optical combining exhibits better carrier-to-noise and distortion ratio (CNDR) with smaller optimum optical modulation index (OMI). Furthermore, the reduced optimum OMI makes the baseband data of optical combining scheme $1 \mathrm{~dB}$ better than that of electrical combining scheme.

\section{Hybrid ANALOG-Digital ModUlator}

Fig. 1 shows a typical hybrid radio channel and digital data transporting link. A single 10-Gb/s baseband data for FTT-x application and multiple wireless signals with carrier around $60 \mathrm{GHz}$ are single sideband (SSB) modulated by a hybrid mode modulator. SSB modulates the radio signal with only one sideband and it is proposed to well combat the fiber dispersion especially in millimeter wave transmission [5]. A 60-GHz reference tone is used to beat with the millimeter wave signals after photodetection and the millimeter wave signals will be down-converted to commercially used wireless frequency band and transmitted in the air link. A remote node with optical filter such as fiber Bragg grating can separate the two signals and feed to their corresponding applications. With sharper optical filter, radio frequency of less than $40 \mathrm{GHz}$ is preferred more for its available commercial optical modulator. Advantages of the link are the enhanced spectra usage, lower infrastructure cost, less laser source, and hence, more convenient to manage. We discuss two possible hybrid analog-digital modulators shown in Fig. 2. Fig. 2(a) shows the electrical combining scheme. This scheme is direct and simple but the radio channels are biased at the nonlinear region due to the existence of digital data. In addition, the analog output efficiency (ac output optical power/ac input electrical power) depends on whether the baseband data is transmitted or not, which might require different optimum operating parameters. Fig. 2(b) shows the optical combining scheme, in which optical power is split and enters two parallel Mach-Zehnder modulators (MZMs) for analog and digital modulation and then combined with an optical coupler. From the plot, it can be seen that both the two MZMs can be biased 




Fig. 1. Typical integrated network including RoF and FTT-x applications.
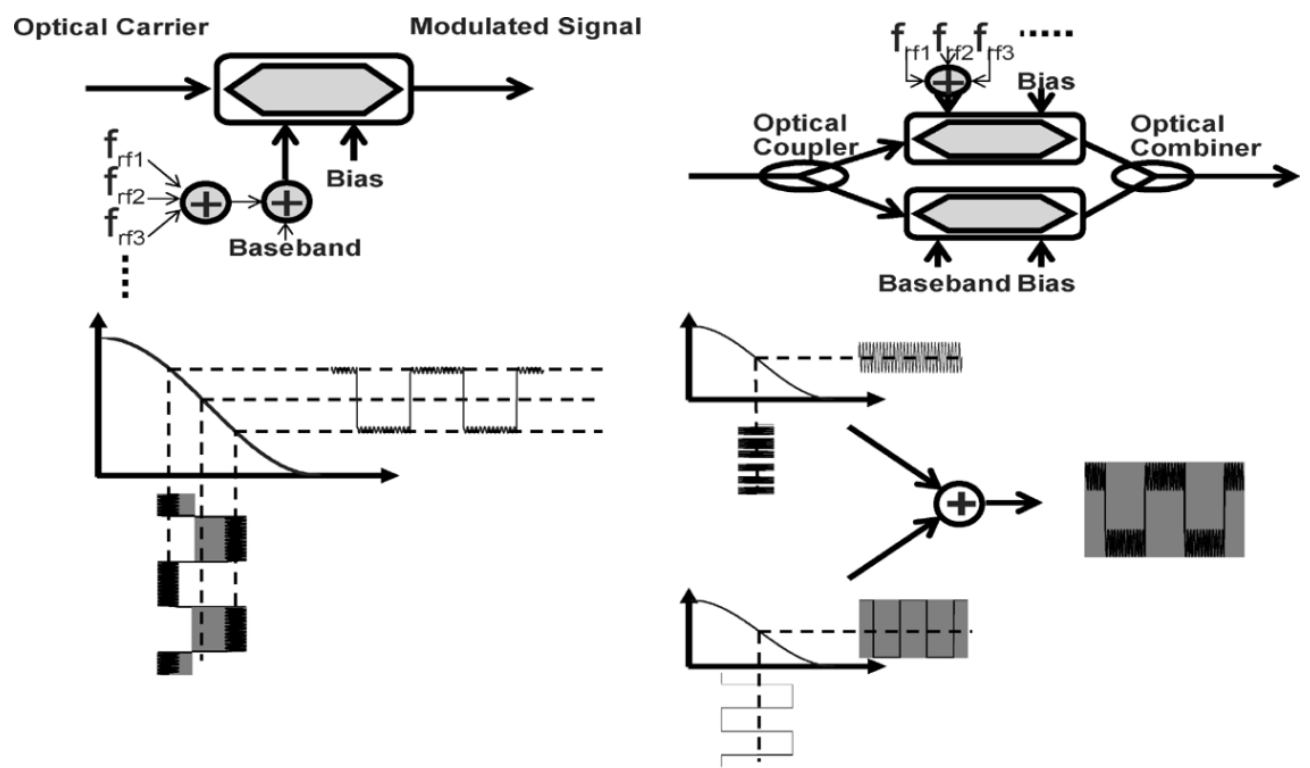

(a)

(b)

Fig. 2. (a) Hybrid mode modulator combines radio and baseband signals in electrical domain. (b) Hybrid mode modulator combines radio and baseband signals in optical domain.

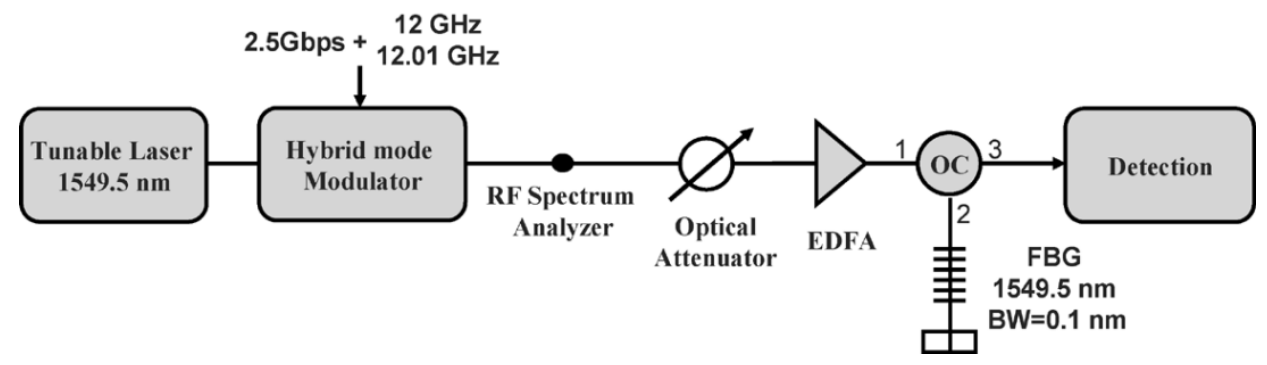

Fig. 3. System setup for simulation and experiment.

at linear region and the radio linearity is independent of the existence of baseband data. However, the requirement of two MZMs, optical coupler, and combiner might make this scheme costly. In addition, the optical combiner will introduce additional 3-dB power loss.

Our simulation and experimental system setup are based on the architecture shown in Fig. 3. A tunable laser operated at a wavelength of $1549.5 \mathrm{~nm}$ is modulated by $2.5-\mathrm{Gb} / \mathrm{s}$ baseband data and two frequency tones at 12 and $12.01 \mathrm{GHz}$ are emulated as two radio channels. The reference beating tone is not included here since two tones are usually enough to evaluate its linearity. The external modulators used are all commercial single-ended MZMs. The input splitting ratio of optical combiner is $40: 60$ for digital and radio signal modulation, respectively, and the radio signal in the upper branch is double sideband modulated. A fiber Bragg grating centered at $1549.5 \mathrm{~nm}$ with 3 -dB bandwidth of 


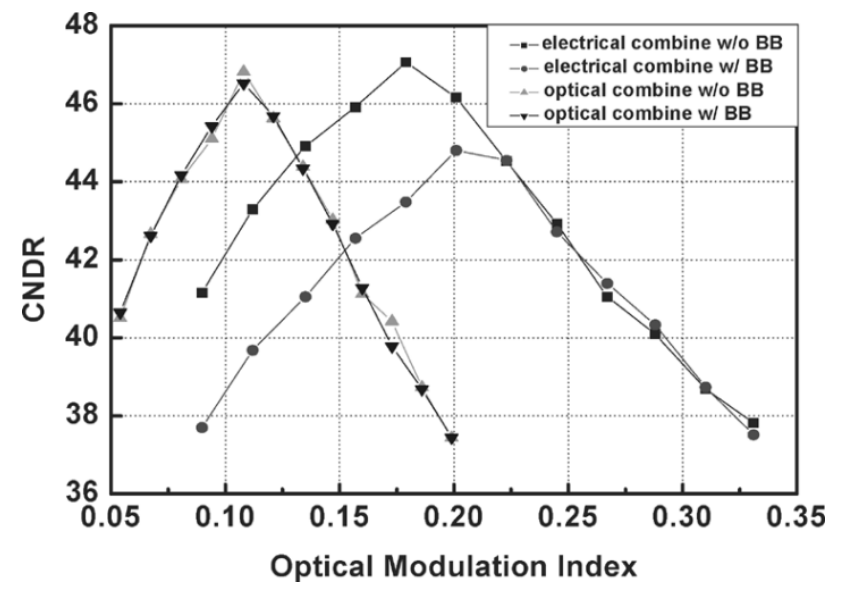

Fig. 4. CNDR of frequency tones at 12 and $12.01 \mathrm{GHz}$ in both schemes.

$0.1 \mathrm{~nm}$ is used to filter out the radio signal and the digital baseband data is detected and bit error counted by a bit-error-rate (BER) tester. CNDR is simulated at the black point in Fig. 3 by simulation software, VPItransmissionMaker 5.0. And BER performance of baseband data is evaluated by experimental results.

\section{SIMULATION AND EXPERIMENTAL RESULTS}

To focus on the impact of interference between radio and baseband signals, the optical power through the radio branch in optical combining scheme is equal to that in electrical combining. Extinction ratio (ER) of the baseband data in both schemes are fixed at $3.6 \mathrm{~dB}$ for fair comparison. This ER is selected for maximal ER in the optical combining method. The CNDR results are shown in Fig. 4. In case of electrical combining, the optimum OMI is reduced and enhanced with baseband data due to the changed biased point of the MZM, while the optical combining scheme completely alleviates this problem. Removal of this interchannel interference is very crucial since it is usually impossible to optimize the system for both cases with or without the transmission of baseband data. Smaller or larger baseband ER would have minimum impact on radio channel in optical combiner, but would not in electrical combiner.

BER of 2.488-Gb/s digital baseband data with two frequency tones is experimentally demonstrated. The used modulation index of analog signals in both schemes are selected from the optimum points of $\mathrm{OMI}=0.11$ and 0.2 , respectively, and baseband ER are hold at $3.6 \mathrm{~dB}$, which is the same as the simulation parameters. The results are shown in Fig. 5. The insets are the optical spectra of the combined signals before and after the fiber Bragg grating. The unwanted frequency tones are rejected by over $10 \mathrm{~dB}$. As expected, the optical combining scheme is $1 \mathrm{~dB}$ better at BER $=10^{-9}$ due to the smaller used optimum OMI. There would be a possibility that both the electrical and optical combiner would be options in access networks having different size and number of channels. The electrical combining scheme is preferred for a smaller area with less radio channels while the

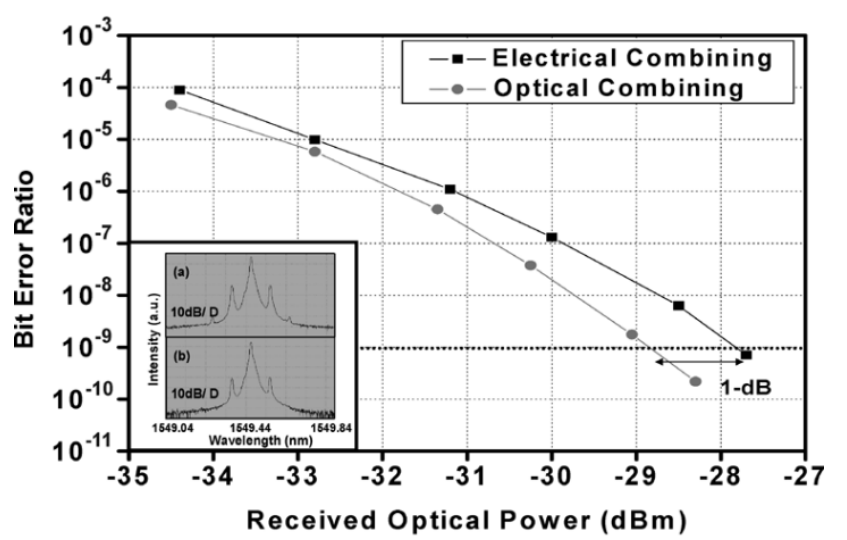

Fig. 5. Measured BER performance of baseband data in both schemes.

optical combining is more suited for a larger area supporting more radio channels. Sensitivity can be further improved if higher ER of baseband data and higher rejection or narrower bandwidth optical filter is used.

\section{CONCLUSION}

An integrated network which combines the radio distribution and digital FTT-x network is imperative needed for its low cost infrastructure. To implement the hybrid analog-digital modulators, two schemes combining signals in electrical and optical domains are proposed and discussed. The simulation results show that the optical combining can reduce the optimum modulation index and avoid the interchannel interference. BER of two schemes with their corresponding optimum modulation index are experimentally verified and due to the smaller OMI of the optical combining method, BER is better than the electrical combining scheme. The major problem to implement the optical combining might be the higher cost of two Mach-Zehnders. However, the cost issue should not be a problem in the near future since the two MZMs can be integrated on a single wafer and will be easier to handle [6].

\section{REFERENCES}

[1] T. Kamisaka, T. Kuri, and K. Kitayama, "Simultaneous modulation and fiber-optic transmission of $10 \mathrm{~Gb} / \mathrm{s}$ baseband and $60 \mathrm{GHz}$ band radio signals on a single wavelength," IEEE Trans. Microw. Theory Tech., pt. 2, vol. 49, no. 10, pp. 2013-2017, Oct. 2001.

[2] K. Ikeda, T. Kuri, and K. Kitayama, "Simultaneous three band modulation and fiber-optic transmission of $2.5 \mathrm{~Gb} / \mathrm{s}$ baseband, microwave-, and $60 \mathrm{GHz}$ band signals on a single wavelength," J. Lightw. Technol., vol. 21, no. 12, pp. 3194-3202, Dec. 2003.

[3] A. Kim, Y. H. Joo, and Y. Kim, "60 GHz wireless communication systems with radio-over-fiber links for indoor wireless LANs," IEEE Trans. Consumer Electron., vol. 50, no. 2, pp. 517-520, May 2004.

[4] C. N. Lo, "A hybrid lightwave transmission system for subcarrier multiplexed video and digital B-ISDN services in the local loop," J. Lightw. Technol., vol. 7, no. 11, pp. 1839-1848, Nov. 1989.

[5] A. Loayssa, C. Lim, A. Nirmalathas, and D. Benito, "Simple optical single-sideband modulator for fiber-radio applications," Electron. Lett., vol. 39, pp. 97-99, 2003.

[6] T. Kawanishi, K. Higuma, T. Fujita, J. Ichikawa, T. Sakamoto, S. Shinada, and M. Izutsu, " $\mathrm{LiNbO}_{3}$ high-speed optical FSK modulator," Electron. Lett., vol. 40, pp. 691-692, 2004. 